\title{
EDUCACIÓN EN IGUALDAD DE GÉNERO PARA PREVENIR LA VIOLENCIA MACHISTA
}

\section{Gender equality education to prevent male violence against women}

\author{
Gabriela Moriana Mateo \\ gabriela.moriana@uv.es \\ Universitat de València - España
}

$\begin{array}{ll}\text { Recibido: } & 12-02-2017 \\ \text { Aceptado: } & 04-05-2017\end{array}$

\section{Resumen}

La violencia contra las mujeres es un fenómeno universal e histórico que afecta a las mujeres y niñas de todas las clases sociales, tratándose de una manifestación de desigualdad entre las mujeres y los hombres. Uno de los ámbitos privilegiados y especialmente importantes desde donde se puede y debe prevenir es la educación. Disponemos de un buen marco conceptual para comprender la complejidad del fenómeno, de datos que indican la gravedad y magnitud del problema y de una normativa de obligado cumplimiento en todos los niveles educativos, porque la igualdad también se aprende y, a pesar de los importantes retos que tiene planteados, la coeducación deviene necesaria para poder erradicar la violencia machista.

Palabras clave: Violencia contra las mujeres, educación, igualdad de oportunidades, coeducación, prevención.

\begin{abstract}
Violence against women is a universal and historical phenomenon that affects women and girls from all social classes, the greatest expression of inequality between women and men. One of the privileged and most important areas from which it can and must be prevent is education. We have a good conceptual framework for understanding the complexity of the phenomenon, data that show the magnitude and how serious problem is, and mandatory regulations that must be followed at all levels of education because equality is also learned and, despite the great challenges involved, coeducation is necessary to enable the eradication of male violence against women.
\end{abstract}

Keywords: Violence against women, education, equal opportunities, coeducation, prevention. 


\section{Introducción}

La problemática de la violencia contra las mujeres empezó a tomar relevancia a partir de la década de los 70 del pasado siglo, gracias al trabajo de las organizaciones de mujeres de todo el mundo que, en su lucha por la igualdad, se fueron dando cuenta de que este tipo de malos tratos no eran casuales ni aislados. Así, iniciaron el proceso de visibilización de esta lacra, llegando a desempeñar un papel primordial en la lucha por su erradicación.

El primer documento internacional que aborda esta cuestión específicamente es la Declaración de la Violencia Contra las Mujeres de Naciones Unidas (ONU, 1994). En ella se afirma que esta problemática está presente en todas las sociedades del mundo, independientemente de su sistema político o económico y que constituye una violación de los derechos humanos y de las libertades fundamentales de las mujeres. Así mismo, reconoce que esta violencia constituye una manifestación de las relaciones de poder históricamente desiguales entre los hombres y las mujeres.

Si bien la violencia contra las mujeres afecta a todas las mujeres, la mencionada Declaración muestra su preocupación por el hecho de que algunos grupos, como, por ejemplo, las pertenecientes a minorías, indígenas, refugiadas, migrantes, habitantes en comunidades rurales o remotas, indigentes, recluidas en instituciones o detenidas, niñas, con discapacidades, ancianas y en situaciones de conflicto armado son particularmente vulnerables. Así mismo, recuerda que en 1990 el Consejo Económico y Social, reconoció que la violencia contra las mujeres en la familia y en la sociedad se ha generalizado y trasciende las diferencias de ingresos, clases sociales y culturas, y debe contrarrestarse con medidas urgentes y eficaces para eliminar su incidencia.

Dada la magnitud y gravedad del problema y atendiendo a las reivindicaciones del movimiento de mujeres, se fueron implementando recomendaciones internacionales, de las que cabe destacar la mencionada Declaración sobre la eliminación de la violencia contra la mujer de la ONU (1994), la Cuarta Conferencia Mundial sobre la Mujer celebrada en Beijing (ONU, 1995) y la Resolución "Prevención de la Violencia: una prioridad de salud pública" de la Organización Mundial de la Salud (OMS, 1996). Así mismo, en el Estado español se aprueba por unanimidad en 2004 la Ley de Medidas de Protección Integral contra la Violencia de Género (Gobierno de España, 2004), en adelante Ley Integral. En ella se afirma que este tipo de violencia no es un problema que afecte al ámbito privado; al contrario, se manifiesta como el símbolo más brutal de la desigualdad existente en nuestra sociedad. Se trata de una violencia que se ejerce contra las mujeres por el hecho mismo de serlo, por ser consideradas, por sus agresores, carentes de los derechos mínimos de libertad, respeto y capacidad de decisión. 
Esta Ley entiende que la violencia de género debe ser abordada de un modo integral y multidisciplinar, empezando por el proceso de socialización y educación. Así, establece medidas de sensibilización e intervención en al ámbito educativo. Pero también, los principios y valores del sistema educativo español, que incluye entre sus fines la formación en el respeto de los derechos y libertades fundamentales y de la igualdad entre hombres y mujeres, así como en el ejercicio de la tolerancia y de la libertad dentro de los principios democráticos de convivencia.

Todas las comunidades autónomas han venido aprobando también sus leyes para regular este tipo de violencia específica contra las mujeres: Castilla La Mancha (2001), Navarra (2002/2015), Canarias (2003), Cantabria (2004), Madrid (2005), Illes Balears (2006), Galicia (2007), Andalucía (2007), Aragón (2007), Murcia (2007-2016), Cataluña (2008), Castilla y León (2010), Extremadura (2011), Principado de Asturias (2011), La Rioja (2011), Comunidad Valenciana (2012). Euskadi, no ha aprobado ley de violencia, pero la incluye en la Ley de Igualdad de Mujeres y Hombres (Gobierno del País Vasco, 2005).

\section{Definición de violencia contra las mujeres}

Dada la complejidad y magnitud del fenómeno de la violencia contra las mujeres, conviene tener en cuenta algunas cuestiones, como las aportaciones de Galtung (2003), que señala tres formas de violencia. En primer lugar, la violencia directa, que es la visible, se concreta en comportamientos y responde a actos de violencia. La segunda, la violencia estructural, se produce un perjuicio en la satisfacción de las necesidades humanas básicas: supervivencia, bienestar, identidad, libertad, etc.

Está originada por todo un conjunto de estructuras, tanto físicas como organizativas, que no permiten la satisfacción de esas necesidades y es la peor de las tres violencias, porque es el origen de todas, la que más mata y a más personas afecta. Y la tercera, la violencia cultural, es una violencia simbólica, que se expresa en infinidad de medios: religión, ideología, lenguaje, arte, ciencia, medios de comunicación, educación, etc. y cumple la función de legitimar la violencia directa y estructural, así como de inhibir o reprimir la respuesta de quienes la sufren.

Seguidamente se representa el triángulo de violencia de Galtung, en el gráfico 1. 


\section{Gráfico 1. Triángulo de violencia de Galtung.}

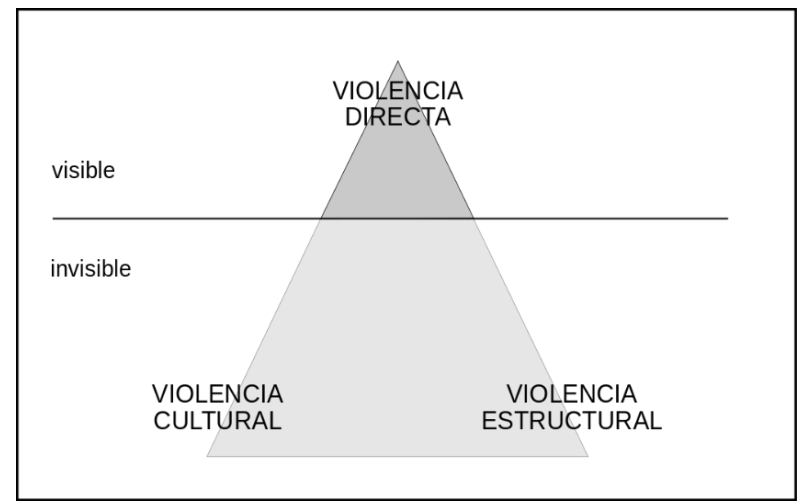

Fuente: Galtung, 2003

Centrándonos en la violencia directa contra las mujeres, la Declaración de la Eliminación de la Violencia (ONU, 1994), entiende todo acto de violencia basado en la pertenencia al sexo femenino que tenga o pueda tener como resultado un daño o sufrimiento físico, sexual o psicológico para las mujeres, así como las amenazas de tales actos, la coacción o la privación arbitraria de la libertad, tanto si se producen en la vida pública como en la vida privada. Así, la violencia contra la mujer abarca los siguientes actos, aunque sin limitarse a ellos:

1. La violencia física, sexual y psicológica que se produzca en la familia, incluidos los malos tratos, el abuso sexual de las niñas en el hogar, la violencia relacionada con la dote, la violación por el marido, la mutilación genital femenina y otras prácticas tradicionales nocivas para las mujeres, los actos de violencia perpetrados por otros miembros de la familia y la violencia relacionada con la explotación.

2. La violencia física, sexual y psicológica perpetrada dentro de la comunidad en general, inclusive la violación, el abuso sexual, el acoso y la intimidación sexuales en el trabajo, en instituciones educacionales y en otros lugares, la trata de mujeres y la prostitución forzada.

3. La violencia física, sexual y psicológica perpetrada o tolerada por el Estado, dondequiera que ocurra.

De manera que, siguiendo la mencionada declaración, podemos hablar de tres tipos de violencia contra las mujeres: física, psíquica y sexual y de tres ámbitos donde se produce: la familia, la comunidad en general y la perpetrada o tolerada por el estado.

Por su parte, la Organización Mundial de la Salud (OMS, 1998), señala distintos tipos de violencia directa contra la mujer a través del ciclo de vida, se expone a continuación, en el cuadro 1. 


\section{Cuadro 1. La violencia contra las mujeres a lo largo de la vida.}

\begin{tabular}{|c|c|}
\hline Fase & Tipo de violencia \\
\hline Pre-natal & $\begin{array}{l}\text { Abortos selectivos según el sexo; efectos sobre el recién nacido de la violencia durante el } \\
\text { embarazo }\end{array}$ \\
\hline Infancia & Infanticidio femenino; abuso físico, sexual y psicológico \\
\hline Niñez & $\begin{array}{l}\text { Matrimonio infantil; mutilación genital femenina; abuso físico, sexual y psicológico; } \\
\text { incesto; prostitución infantil y pornografía }\end{array}$ \\
\hline $\begin{array}{l}\text { Adolescencia } \\
\text { y vida adulta }\end{array}$ & $\begin{array}{l}\text { Violencia durante el cortejo y el noviazgo (alteración de bebidas y violaciones); sexo } \\
\text { forzado por razones económicas (niñas estudiantes que tienen relaciones sexuales con } \\
\text { adultos a cambio de favores); incesto; abuso sexual en el sitio de trabajo; violaciones; } \\
\text { acosamiento sexual; prostitución y pornografía forzada; tráfico de mujeres; violencia } \\
\text { conyugal; violación marital; abuso y homicidio; homicidio conyugal; abuso psicológico; } \\
\text { abuso de mujeres discapacitadas; embarazos forzados }\end{array}$ \\
\hline Vejez & $\begin{array}{l}\text { "Suicidio" forzado u homicidio de viudas por razones económicas; abuso físico, sexual y } \\
\text { psicológico }\end{array}$ \\
\hline
\end{tabular}

Fuente: Organización Mundial de la Salud, 1998

Por otra parte, y centrándonos en el ámbito estatal, la Ley Integral tiene por objeto actuar contra la violencia que, como manifestación de la discriminación, la situación de desigualdad y las relaciones de poder de los hombres sobre las mujeres, se ejerce sobre éstas por parte de quienes sean o hayan sido sus cónyuges o de quienes estén o hayan estado ligados a ellas por relaciones similares de afectividad, aun sin convivencia.

La violencia de género a que se refiere esta ley comprende todo acto de violencia física y psicológica, incluida las agresiones a la libertad sexual, las amenazas, las coacciones o la privación arbitraria de libertad. Es decir, la violencia física, psíquica y sexual, y las esferas o ámbitos en los que se produce, tanto en el espacio público como en el privado.

Aunque esta ley habla de violencia de género, probablemente uno de los tipos o manifestaciones más importantes, no contempla todos los supuestos de violencia contra las mujeres, porque como hemos visto, la reduce al ámbito único y exclusivo de la pareja. Esto no sólo genera confusión, porque identifica violencia de género con un sólo tipo de violencia contra las mujeres, sino que invisibiliza la definición que se da en el plano internacional. Por lo tanto, la ley española deja fuera la mayor parte de los supuestos que son considerados violencia contra las mujeres.

No ocurre lo mismo en muchas Comunidades Autónomas, donde se ha optado por definiciones más amplias. Asimismo, algunas autonomías, como es el caso de Andalucía, País Vasco y Comunidad Valenciana también han incluido la violencia económica. Así, a los efectos de la Ley de Violencia Integral de la Comunidad Valenciana (Gobierno de la Comunidad 
Valenciana, 2012), se considera toda aquella limitación, privación no justificada legalmente o discriminación en la disposición de sus bienes, recursos patrimoniales o derechos económicos, comprendidos en el ámbito de convivencia de la pareja o en los casos de ruptura de la relación.

En referencia a la violencia directa en las relaciones de pareja, Walker descubrió, a partir de su trabajo con mujeres en 1979, que la violencia de género, sobre todo física, no suele comenzar repentinamente; sino que responde a un proceso que mantiene a la víctima encerrada en él, al que denominó el Ciclo de la Violencia, éste tiene tres fases, se representa seguidamente, en el gráfico 2.

\section{Gráfico 2. Ciclo de la violencia}

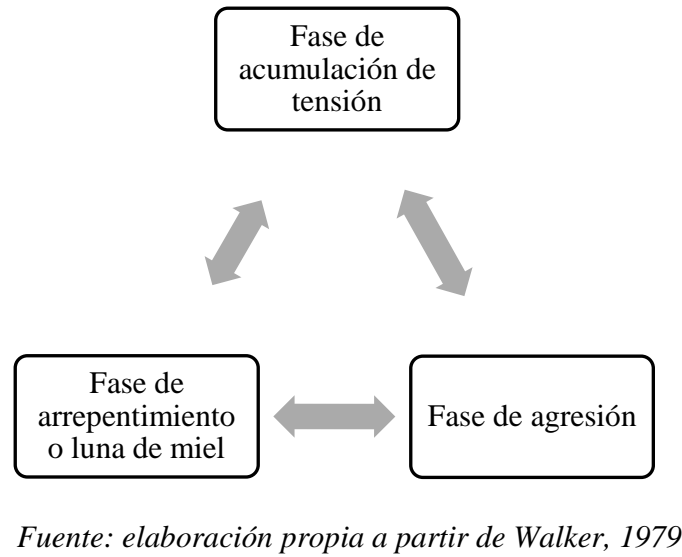

1. Fase de acumulación de tensión, las tensiones se van creando. Estas tensiones se establecen a partir de pequeños incidentes y conflictos. El agresor va volviéndose más agresivo, encontrando motivos de conflicto en cada situación.

2. Fase de agresión, es la de los episodios agudos, la explosión. El agresor pasa a la acción, la violencia verbal y psicológica están presentes, surgen las amenazas y ante cualquier mínimo incidente, se produce el acto de agresión física. Suele ser en esta etapa cuando las mujeres que sufren violencia buscan ayuda.

3. Fase de arrepentimiento o luna de miel. El arrepentimiento del agresor se expresa a través de comportamientos de reconciliación. Este aparente arrepentimiento hace surgir en las mujeres la esperanza de que no volverá a pasar, de que no lo volverá a hacer si ella accede a sus demandas. Tras esta etapa, volverán los conflictos y el ciclo comienza de nuevo.

Los ciclos se dan en procesos cada vez más cortos de tiempo a medida que se repiten las conductas violentas, los ataques cada vez son más frecuentes, intensos y peligrosos, el ciclo de la violencia es imparable. Algunas autoras incluso creen que a lo largo del tiempo la última fase va desapareciendo y sólo se dan las dos primeras (Cerezo, 2000; Ruiz-Jarabo y Blanco, 2005). La 
comprensión de que se trata de un ciclo permite comprender por qué muchas mujeres al ser agredidas buscan apoyo, protección y denuncian la situación y, no obstante, en muchas ocasiones, vuelven con el maltratador o no lo abandonan y retiran la denuncia. Será después de muchas ocasiones de ese volver a comenzar cuando las mujeres decidan romper con la relación violenta, cada una tiene su momento, dependiendo de su situación y circunstancias.

\section{De qué hablamos: algunos datos sobre violencia machista}

Según la investigación del Ministerio de Sanidad, Política Social e Igualdad (2010), el 13,1\% de los chicos reconoce haber ejercido o intentado situaciones de maltrato respecto a las chicas con las que salían en alguna ocasión, y el 9,2\% de las chicas haber vivido situaciones de maltrato. Un $26 \%$ de los chicos considera que no es maltrato "controlar las relaciones de pareja", un 30,7\% de que no es maltrato "decirle que no vale nada", y hasta un $35 \%$ de ellos considera que no es maltrato "controlar todo lo que hace" (afirmación que secunda el 26,2\% de las chicas). En cuanto a los patrones de dominio-sumisión, se encuentra que el $12,2 \%$ de los chicos se mostraba de acuerdo con la afirmación "para tener una buena relación de pareja es deseable que la mujer evite llevar la contraria al hombre". Si hacemos referencia a la violencia física manifiesta, el 6,8\% de los chicos está de acuerdo con que cuando una mujer es agredida por su marido, algo habrá hecho ella para provocarlo.

Respecto a la violencia alguna vez en la vida, según la Macroencuesta de Violencia Contra las Mujeres de la Delegación del Gobierno para la Violencia de Género $(2015)^{1}$, el 11,6\% de las mujeres residentes en el Estado español de 16 o más años ha sufrido violencia física de alguna persona con la que no mantiene ni ha mantenido una relación de pareja. El 7,2\% de las mujeres residentes en el Estado español de 16 o más años ha sufrido violencia sexual de alguna persona con la que no mantiene ni ha mantenido una relación de pareja. Un 12,5\% de mujeres residentes en el Estado español de 16 o más años ha sufrido violencia física y/o sexual por parte de su pareja o ex pareja. El 10,3\% violencia física y el 8,1\% violencia sexual. El 25,4\% de las mujeres residentes en España de 16 o más años han sufrido violencia psicológica de control, el 21,9\% violencia psicológica emocional y el 10,8\% violencia económica de alguna pareja o expareja.

Por su parte, siguiendo datos de la Delegación del Gobierno para la Violencia de Género, el año 2016 han sido asesinadas en el estado español 44 mujeres por sus parejas o exparejas (solo 13 habían presentado denuncia), 60 el año 2015 y más de 830 desde 2003. Así, en el año 2016, 26 menores de 18 años se han quedado huérfanos a causa de la violencia de género. De acuerdo con la misma fuente, el año 2015, denunciaron violencia de género 129.193 mujeres, aunque

\footnotetext{
${ }^{1}$ La Macroencuesta de Violencia contra la Mujer 2015, se ha realizado en colaboración con el Centro de Investigaciones Sociológicas (CIS) a una muestra de 10.171 mujeres de 16 y más años, representativas de la población femenina residente en España.
} 
según la Macroencuesta de Violencia de la Delegación del Gobierno (2015), siete de cada diez (el 67,8\%) nunca la han denunciado.

Finalmente y respecto a las mujeres europeas y violencia contra las mujeres y de género, los datos de la Encuesta Europea de la Agencia de los Derechos Fundamentales de la Unión Europea $(2014)^{2}$, revelan que, una de cada tres europeas de entre 18 y 74 años de edad ha sufrido violencia física o sexual desde los 15 años, lo que equivale a 62 millones de mujeres. El 22\% de las mujeres han experimentado violencia física o sexual por su parte de su pareja masculina. El 5\% de las europeas mayores de 15 años (una de cada 20) han sido violadas. En los casos en los que la pareja no era el agresor, una de cada diez violadas indican que más de un hombre estuvo implicado. Sólo una de cada tres mujeres que sufrieron agresiones físicas por parte de su pareja denunció el caso más grave a la policía o a otra organización. Cuando el agresor no era su pareja, sólo una de cada cuatro mujeres informó de ello. Una de cada cuatro mujeres que fueron víctimas de una agresión sexual no fue a la policía por vergüenza. El 43\% de las mujeres ha sufrido alguna forma de violencia psicológica por parte de su actual o anterior pareja masculina.

\section{Legislación estatal contra la violencia de género desde la educación}

\subsection{Medidas de protección integral}

Siguiendo la Ley Integral, el sistema educativo español incluirá, dentro de sus principios de calidad, la eliminación de los obstáculos que dificultan la plena igualdad entre hombres y mujeres y la formación para la prevención de conflictos y para la resolución pacífica de los mismos. Así establece los objetivos según el nivel formativo. La Educación Infantil contribuirá a desarrollar en la infancia el aprendizaje en la resolución pacífica de conflictos. La Educación Primaria contribuirá a desarrollar en el alumnado su capacidad para adquirir habilidades en la resolución pacífica de conflictos y para comprender y respetar la igualdad entre sexos. La Educación Secundaria Obligatoria contribuirá a desarrollar en el alumnado la capacidad para relacionarse con los demás de forma pacífica y para conocer, valorar y respetar la igualdad de oportunidades de hombres y mujeres. El Bachillerato y la Formación Profesional contribuirán a desarrollar en el alumnado la capacidad para consolidar su madurez personal, social y moral, que les permita actuar de forma responsable y autónoma y para analizar y valorar críticamente las desigualdades de sexo y fomentar la igualdad real y efectiva entre hombres y mujeres. La Enseñanza para las personas adultas incluirá entre sus objetivos desarrollar actividades en la resolución pacífica de conflictos y fomentar el respeto a la dignidad de las personas y a la igualdad entre hombres y mujeres. Las Universidades

\footnotetext{
${ }^{2}$ Los resultados de la encuesta de la Agencia de Derechos Fundamentales de la Unión Europea (FRA) sobre la violencia contra la mujer se basan en entrevistas personales realizadas a 42.000 mujeres en los 28 Estados miembros de la Unión Europea, con una media de 1.500 entrevistas por país. Los resultados son representativos de las experiencias y las opiniones de mujeres con edades entre los 18 y los 74 años residentes en la Unión Europea.
} 
incluirán y fomentarán en todos los ámbitos académicos la formación, docencia e investigación en igualdad de género y no discriminación de forma transversal.

Por su parte, con el fin de garantizar la efectiva igualdad entre hombres y mujeres, las administraciones educativas velarán para que en todos los materiales educativos se eliminen los estereotipos sexistas o discriminatorios y para que fomenten el igual valor de hombres y mujeres. Así mismo, las administraciones educativas adoptarán las medidas necesarias para que en los planes de formación inicial y permanente del profesorado se incluya una formación específica en materia de igualdad, con el fin de asegurar que adquieren los conocimientos y las técnicas necesarias que les habiliten para:

1. La educación en el respeto de los derechos y libertades fundamentales y de la igualdad entre hombres y mujeres y en el ejercicio de la tolerancia y de la libertad dentro de los principios democráticos de

convivencia.

2. La educación en la prevención de conflictos y en la resolución pacífica de los mismos, en todos los ámbitos de la vida personal, familiar y social.

3. La detección precoz de la violencia en el ámbito familiar, especialmente sobre la mujer y los hijos e hijas.

4. El fomento de actitudes encaminadas al ejercicio de iguales derechos y obligaciones por parte de mujeres y hombres, tanto en el ámbito público como privado, y la corresponsabilidad entre los mismos en el ámbito doméstico.

Pero, además, se adoptarán las medidas precisas para asegurar que los Consejos Escolares impulsen la adopción de medidas educativas que fomenten la igualdad real y efectiva entre hombres y mujeres. Con el mismo fin, en el Consejo Escolar del Estado se asegurará la representación del Instituto de la Mujer y de las organizaciones que defiendan los intereses de las mujeres, con implantación en todo el territorio nacional.

Finalmente, los servicios de inspección educativa velarán por el cumplimiento y aplicación de los principios y valores recogidos en este capítulo en el sistema educativo destinados a fomentar la igualdad real entre mujeres y hombres.

\subsection{Medidas para la igualdad efectiva}

De acuerdo con la Ley Orgánica 3/2007, de 22 de marzo, para la igualdad efectiva de mujeres y hombres, el sistema educativo incluirá entre sus fines la educación en el respeto de los derechos y libertades fundamentales y en la igualdad de derechos y oportunidades entre mujeres y hombres. Así, el sistema educativo incluirá, dentro de sus principios de calidad, la eliminación de los 
obstáculos que dificultan la igualdad efectiva entre mujeres y hombres y el fomento de la igualdad plena entre unas y otros.

Para integración del principio de igualdad en la política de educación, las administraciones educativas garantizarán un igual derecho a la educación de mujeres y hombres a través de la integración activa, en los objetivos y en las actuaciones educativas, del principio de igualdad de trato, evitando que, por comportamientos sexistas o por los estereotipos sociales asociados, se produzcan desigualdades entre mujeres y hombres. Las administraciones educativas, en el ámbito de sus respectivas competencias, desarrollarán, con tal finalidad, las siguientes actuaciones:

1. La atención especial en los currículos y en todas las etapas educativas al principio de igualdad entre mujeres y hombres.

2. La eliminación y el rechazo de los comportamientos y contenidos sexistas y estereotipos que supongan discriminación entre mujeres y hombres, con especial consideración a ello en los libros de texto y materiales educativos.

3. La integración del estudio y aplicación del principio de igualdad en los cursos y programas para la formación inicial y permanente del profesorado.

4. La promoción de la presencia equilibrada de mujeres y hombres en los órganos de control y de gobierno de los centros docentes.

5. La cooperación con el resto de las administraciones educativas para el desarrollo de proyectos y programas dirigidos a fomentar el conocimiento y la difusión, entre las personas de la comunidad educativa, de los principios de coeducación y de igualdad efectiva entre mujeres y hombres.

6. El establecimiento de medidas educativas destinadas al reconocimiento y enseñanza del papel de las mujeres en la historia.

En el ámbito de la educación superior, las administraciones públicas en el ejercicio de sus respectivas competencias fomentarán la enseñanza y la investigación sobre el significado y alcance de la igualdad entre mujeres y hombres. En particular, y con tal finalidad, promoverán:

1. La inclusión, en los planes de estudio en que proceda, de enseñanzas en materia de igualdad entre mujeres y hombres.

2. La creación de postgrados específicos.

3. La realización de estudios e investigaciones especializadas en la materia. 


\section{Educación para la igualdad: la escuela coeducativa}

Siguiendo el Plan Director para la coeducación y la prevención de la violencia de género en el sistema educativo del Gobierno del País Vasco (2013), en el sistema educativo ha habido grandes avances, hoy en día las mujeres son mayoría en los niveles postobligatorios de la enseñanza, la enseñanza mixta se ha generalizado como modelo en la enseñanza pública y en la casi totalidad de los centros concertados o privados. Además, desde un amplio marco normativo se insta al sistema educativo a trabajar para una sociedad igualitaria desde la escuela. Estos hechos han reforzado la creencia de que la igualdad de oportunidades de mujeres y hombres se ha alcanzado en el ámbito educativo. El espejismo se ve fortalecido en la medida en que los resultados de las chicas son superiores, su rendimiento académico en todas las etapas es mejor, su tasa de fracaso y abandono escolar es inferior y su acceso a las carreras reconocidas tradicionalmente como masculinas es cada vez mayor.

Sin embargo, las relaciones entre niñas y niños, chicas y chicos siguen siendo asimétricas y se dan numerosas situaciones basadas en el abuso. Las capacidades, habilidades y proyectos de vida siguen estando ligadas al género. En los contenidos curriculares las aportaciones de las mujeres no están visibilizadas, y el sexismo y la violencia de género son realidades que están incidiendo en el desarrollo de la vida de adolescentes y jóvenes, por lo que es necesario seguir profundizando en la educación en igualdad o mejor, en la coeducación.

La escuela coeducativa enfatiza el reconocimiento de las mujeres en todo aquello que hace referencia a la organización y la gestión del sistema educativo y de los centros escolares, la relación y la interacción entre el alumnado y el profesorado, los currículums, el lenguaje, las unidades de aprendizaje, los materiales, los libros, etc. La coeducación trata de adoptar todas las medidas apropiadas para modificar las pautas sociales y culturales de comportamiento de los hombres y de las mujeres y eliminar las creencias y las prácticas basadas en la idea de la inferioridad o la superioridad de uno de los sexos y en la atribución de papeles estereotipados a los hombres y a las mujeres.

Promover la igualdad de mujeres y hombres, y prevenir la violencia de género implica necesariamente seguir avanzando en la transformación de la actual escuela mixta en una escuela coeducativa, una escuela que tenga como referencia a las personas y el desarrollo de cada una de sus identidades, una escuela más justa para todas las personas, donde las diferencias existentes no supongan desigualdades de género, educativas y sociales.

La coeducación supone educar desde la igualdad, es un método de intervención educativo cuyas bases se asientan en el reconocimiento de las potencialidades e individualidades de niñas y niños, independientemente de su sexo. Se trata de buscar el desarrollo integral de la persona al margen de los estereotipos y roles en función del sexo, y de rechazar cualquier forma de discriminación y de violencia contra las mujeres. La guía de Coeducación del Instituto de la Mujer 
(2008), presenta las características de los modelos de escuela en relación a la Igualdad de Oportunidades entre mujeres y hombres, se expone seguidamente, en el cuadro 2.

\section{Cuadro 2. Características de los modelos de escuela en relación a la Igualdad de Oportunidades entre mujeres y hombres}

\begin{tabular}{|c|c|c|c|c|}
\hline $\begin{array}{l}\text { Sistema } \\
\text { cultural }\end{array}$ & Valores & Normas & Legitimación & $\begin{array}{l}\text { Conocimiento } \\
\text { Empírico }\end{array}$ \\
\hline $\begin{array}{l}\text { Escuela de } \\
\text { Roles } \\
\text { Separados }\end{array}$ & $\begin{array}{l}\text { Educación para el } \\
\text { rol sexual. } \\
\text { Asignación de } \\
\text { géneros. } \\
\text { Socialización de } \\
\text { cada grupo sexual } \\
\text { en los ámbitos } \\
\text { público y privado }\end{array}$ & $\begin{array}{l}\text { Separación física } \\
\text { de los procesos } \\
\text { educativos }\end{array}$ & $\begin{array}{l}\text { Creencia en la } \\
\text { superioridad } \\
\text { masculina }\end{array}$ & $\begin{array}{l}\text { Fundamentos de } \\
\text { tipo moral, por lo } \\
\text { que su análisis } \\
\text { empírico no } \\
\text { resulta relevante }\end{array}$ \\
\hline $\begin{array}{l}\text { Escuela } \\
\text { Mixta }\end{array}$ & $\begin{array}{l}\text { Educación igual } \\
\text { para niños y niñas } \\
\text { como } \\
\text { ciudadanos/as de } \\
\text { una sociedad } \\
\text { democrática }\end{array}$ & $\begin{array}{l}\text { Acento en el } \\
\text { individualismo sin } \\
\text { distinción por } \\
\text { género. Igualdad } \\
\text { en el acceso a los } \\
\text { recursos }\end{array}$ & $\begin{array}{l}\text { Meritocracia. } \\
\text { Premio en } \\
\text { función } \\
\text { de los méritos y } \\
\text { rendimientos } \\
\text { individuales } \\
\text { dirigidos a ser } \\
\text { valorados desde } \\
\text { el } \\
\text { punto de vista } \\
\text { productivo }\end{array}$ & $\begin{array}{l}\text { Eficacia para el } \\
\text { individuo y para la } \\
\text { convivencia social, } \\
\text { existen } \\
\text { estrategias } \\
\text { alternativas y se } \\
\text { rechaza la escuela } \\
\text { separada de la } \\
\text { socialización }\end{array}$ \\
\hline $\begin{array}{l}\text { Escuela } \\
\text { Coeducativa }\end{array}$ & $\begin{array}{l}\text { Educación dirigida } \\
\text { a la eliminación } \\
\text { de los } \\
\text { estereotipos por } \\
\text { razón de sexo } \\
\text { eliminando } \\
\text { situaciones de } \\
\text { desigualdad y } \\
\text { jerarquías } \\
\text { culturales } \\
\text { sexistas }\end{array}$ & $\begin{array}{l}\text { Atención } \\
\text { específica a las } \\
\text { necesidades de } \\
\text { cada grupo } \\
\text { (currículo, } \\
\text { integración con el } \\
\text { profesorado, } \\
\text { controles de } \\
\text { género en todos } \\
\text { los ámbitos del } \\
\text { sistema escolar, } \\
\text { etc.) }\end{array}$ & $\begin{array}{l}\text { La escuela no } \\
\text { debe ser una } \\
\text { institución } \\
\text { reproductora de } \\
\text { desigualdades, } \\
\text { debe ser una } \\
\text { institución de } \\
\text { reconocimiento } \\
\text { de } \\
\text { la diferencia }\end{array}$ & $\begin{array}{l}\text { Basado en la } \\
\text { sociología de la } \\
\text { educación a } \\
\text { través del } \\
\text { conocimiento de } \\
\text { las especificidades } \\
\text { de ambos grupos } \\
\text { sexuales y de las } \\
\text { distintas } \\
\text { expresiones de } \\
\text { sexismo }\end{array}$ \\
\hline
\end{tabular}

Fuente: Instituto de la Mujer, 2008. 


\section{El reto de la coeducación para prevenir la violencia contra las mujeres}

La importancia y la complejidad de la tarea de transformar los valores sexistas hacia valores de igualdad de mujeres y hombres y la urgencia de prevenir la violencia de género desde el ámbito educativo, hace necesario resituarse en la tarea de coeducar. Esto supone, reconocer cuáles han sido los avances, identificar cuáles son los déficits que persisten en materia de coeducación y definir también los nuevos retos que tiene la comunidad educativa.

El trabajo para abordarlos debe plantearse de forma integrada, en todos los aprendizajes escolares y con la implicación de toda la comunidad educativa. Por ello, y siguiendo el Plan Director para la coeducación y la prevención de la violencia de género en el sistema educativo del Gobierno del País Vasco (2013), la comunidad educativa tiene importantes retos:

1. Ahondar en el modelo de escuela coeducativa e introducir la perspectiva de género en la cultura y en las prácticas de los centros desde un planteamiento integral, para ello será necesario:

- La revisión del currículum desde la perspectiva de género. Tanto el currículum oficial que se relaciona con las materias que se enseñan en los centros educativos, como el currículum oculto, pues tienen una incidencia fundamental en la socialización de género de las niñas y los niños. El currículo explícito menciona como importantes los hechos, los conocimientos, las experiencias de los hombres, quedando excluida e invisibilizada la experiencia y el saber de las mujeres en las materias escolares. Por tanto, la herencia cultural que se transmite continúa siendo androcéntrica. Además, los mensajes que se dan en la escuela de manera implícita jerarquizan los valores considerados socialmente masculinos sobre los femeninos. El currículum basado en el desarrollo de competencias abre la oportunidad para trabajar, valorar y evaluar más allá de lo cognitivo, aspectos que se consideran fundamentales para la educación integral del alumnado.

- Potenciar la elección de libros de textos y materiales didácticos inclusivos y coeducativos. Los libros de texto y materiales en general describen un tipo de realidad social, unas formas de organización, unos valores, unas maneras de expresar las emociones, unas normas, unos modelos de sociedades, de vida, de familias y de relaciones basadas en estereotipos de género que no representan toda la realidad social actual. Es importante que los libros ofrezcan, tanto a chicos como a chicas, modelos de identificación que no limiten sus expectativas, en ningún caso, por razones de sexo. Las editoriales se han adaptado a las exigencias de la ley, que rechaza toda discriminación por razón de sexo, y es constatable lo mucho que se ha avanzado desde aquellos textos que establecían claras discriminaciones entre hombres y mujeres, tanto por las 
imágenes que ofrecían, como por el número de apariciones, el protagonismo de los personajes, etc. Sin embargo, como indica el estudio de Pérez y Gargallo (n.d.), cuando se analizan los libros de texto en profundidad, se puede comprobar que la discriminación todavía existe, aunque se produce de una manera mucho más sutil.

- Capacitar, para ser conscientes sobre las diferentes expectativas que persisten respecto al alumnado. Es necesario reconocer la socialización diferenciada y sexista, para poder identificar cuándo está limitando las actitudes, expectativas vitales y profesionales, capacidades, etc. Todas estas expectativas diferenciadas, deben emerger del plano inconsciente al consciente para posibilitar la intervención. Para ello, es imprescindible la formación permanente y progresiva en materia de coeducación de las diferentes personas implicadas de la comunidad educativa

- Incluir el análisis de género en el uso de los espacios. Así, entre otras cuestiones, se revela que en los patios y las zonas de recreo la distribución del espacio sigue siendo sexista y la jerarquía de los juegos, las actitudes de las niñas y niños o la falta de organización espacial del patio suele situar a los niños en el espacio central y arrinconar a las niñas.

- Revisar la evaluación desde la perspectiva de género. Las pruebas y textos utilizados en la evaluación han recibido las mismas críticas que los materiales de lectura: predominio de escenarios y personajes masculinos, tratamiento frívolo de las mujeres y lenguaje e ilustraciones sexistas. Otro de los aspectos a destacar es que existe una tendencia a otorgar calificaciones más bajas a las chicas/mujeres y más altas a los chicos/hombres cuando se conoce el sexo de la persona a calificar, lo que en algunos países ha llevado a introducir el anonimato (de nombre y sexo) del alumnado (Agencia Ejecutiva en el Ámbito Educativo y Cultural, 2010).

- Implicar a las familias en la tarea de coeducar. Los aprendizajes se realizan en diferentes contextos, por lo que es necesario buscar coherencia y continuidad entre los mismos, la colaboración de las familias es fundamental en la tarea de educar. Por ello es necesario aumentar la participación activa de las madres y padres en la educación y en la escuela.

2. Orientar al alumnado a elegir su itinerario vital, académico y profesional desde la libertad, la diversidad de opciones y sin condicionamientos de género. A pesar de los avances de estas últimas décadas, las elecciones personales, profesionales y vitales de niñas y niños siguen estando marcadas por los estereotipos de género. El género es determinante en las distintas elecciones vitales, profesionales y personales del alumnado. Es necesario intervenir en este proceso desde todas las vías: asignaturas, mensajes explícitos e implícitos del profesorado, familias, etc. La tutoría también puede ser un espacio privilegiado para hacer consciente al 
alumnado sobre los estereotipos de género que persisten y orientar para desarticular creencias y prejuicios sexistas que pueden estar incidiendo en sus decisiones.

3. Integrar la perspectiva de género en el abordaje del fracaso escolar y garantizar que el acceso al sistema educativo sea universal, y la continuidad en éste para todas las niñas y niños, teniendo en cuenta la diversidad del alumnado (étnica, cultural, social, económica, funcional, sexual, etc.). Estas actitudes y el fracaso escolar no suelen relacionarse desde el mundo educativo con la variable género y, sin embargo, es crucial tener en cuenta el modelo de masculinidad en el que son socializados los chicos, así como el modelo de feminidad en el que lo son las chicas para entender sus diferentes actitudes en el aula, y sus expectativas con respecto a su trayectoria académica y vital.

4. Educar para unas relaciones afectivo-sexuales y una convivencia basada en la diversidad sexual y en la igualdad de mujeres y hombres. La preocupación por la educación sexual persiste en el ámbito educativo ya que, pese a los avances, se constata que las relaciones afectivas y sexuales entre jóvenes siguen atravesadas en gran medida por el sexismo.

5. Prevenir la violencia de género, detectar de forma precoz los casos y prestar una atención eficaz a las víctimas, implicando de manera especial a toda comunidad educativa. Desde todos los sectores sociales preocupados por la incidencia de la violencia de género en la juventud se identifica al sector educativo como un agente clave en la prevención de ésta.

En el marco preventivo, algunos de los aspectos a tener en cuenta son:

1. El sexismo como factor explicativo de los conflictos y del maltrato entre iguales en las aulas. El modelo de masculinidad tradicional en el que se socializan los chicos, favorece e impulsa una resolución violenta de los conflictos y el aprendizaje en el ejercicio del poder y la violencia.

2. Las microviolencias. Todavía existe desconocimiento y, por tanto, tolerancia, con aquellas violencias que no son tan perceptibles, por lo que las acciones contra ellas son casi inexistentes, pese al gran malestar y daño que producen.

3. Los modelos de relación y de atracción. A menudo, la atracción y el deseo en la elección de las relaciones afectivas y sexuales se relacionan con la dominación, el control y el sufrimiento, y se separan totalmente el amor y la pasión. La atracción por personas posesivas y la identificación de los celos con el amor conducen a la aceptación de la dominación, a la imposición de la autoridad y a la renuncia de elementos importantes de la propia vida y el espacio personal en las chicas. 
En el marco de la detección precoz para la atención eficaz, existen algunas situaciones y o colectivos en los que la detección precoz es aún más urgente, en la medida en que ésta nos permitirá atender e intervenir en los casos y evitar así los daños que se produzcan. Algunas de las situaciones y/o colectivos con mayor vulnerabilidad son las hijas e hijos de mujeres que sufren violencia de género, las niñas en riesgo de mutilación genital femenina y las niñas y adolescentes con necesidades educativa especiales.

\section{A modo de conclusiones}

Como hemos visto, disponemos de un importante marco teórico y conceptual para comprender la complejidad del fenómeno de la violencia contra las mujeres. Así como, de los datos que nos indican la magnitud y gravedad del problema. Pero, además, contamos con una significativa normativa internacional y estatal, de obligado cumplimiento, en todos los niveles educativos.

Porque la escuela puede y debe contribuir a transformar las relaciones de poder, resultado de un sistema social sexista e injusto que convierte las diferencias biológicas en desigualdades. Para ello, se tienen que realizar los cambios necesarios que aseguren una socialización igualitaria en el ámbito educativo.

La educación en igualdad deviene imprescindible para ir avanzando en la transformación de un modelo de escuela mixta a un modelo de escuela coeducativa que contribuya a prevenir la violencia machista.

A pesar de los importantes retos que tiene planteados, la coeducación se ha ido abriendo paso durante los últimos años en el sistema educativo español a partir de diversas experiencias de educación para la igualdad. Sin embargo, estas prácticas han detectado ámbitos del proceso educativo y de sus resultados en los que se pone de manifiesto que, hoy por hoy, en la escuela, todavía perduran elementos que reproducen y perpetúan el sexismo y la desigualdad entre los hombres y mujeres que formaran nuestra sociedad.

Como ya se ha dicho y para finalizar, la violencia contra las mujeres es la expresión más brutal de esa desigualdad. La escuela es un espacio privilegiado para interiorizar y aprender nuevos modelos más igualitarios y respetuosos.

La lucha por la eliminación de la violencia contra las mujeres necesita una actuación preventiva desde el inicio del ciclo educativo, en todos los ámbitos y fases y desde una perspectiva integral. Porque la igualdad también se aprende y las mujeres y las niñas tienen derecho a la igualdad real y una vida libre de violencias machistas. Se trata de una cuestión de justicia social y de derechos humanos, de los derechos humanos de la mitad población, las mujeres y las niñas. 


\section{BIBLIOGRAFÍA}

- Agencia de Derechos Fundamentales de la Unión Europea (FRA) (2014): "Violencia de género contra las mujeres: una encuesta a escala de la UE". Resumen de conclusiones. Luxemburgo. Disponible en: http://www.lrmcidii.org/wp-content/uploads/2014/03/fra-2014vaw-survey-main-results_en1.pdf [01/02/2017].

- Agencia Ejecutiva en el Ámbito Educativo y Cultural (2010): "Diferencias de género en los resultados educativos: medidas adoptadas y situación actual en Europa”. Bruselas: P9 Eurydice. Disponible en: http://eacea.ec.europa.eu/education/Eurydice/documents/thematic_reports/120ES.pdf [10/02/2017].

- Cerezo, Ana Isabel (2000): El homicidio en la pareja: tratamiento criminológico, Criminología y educación social. Valencia: Tirant lo Blanch.

- Delegación del Gobierno para la Violencia de Género (2013): VII Informe anual del Observatorio Estatal de Violencia sobre la Mujer. Madrid: Gobierno de España. Versión [online] Disponible

en: http://www.violenciagenero.msssi.gob.es/violenciaEnCifras/observatorio/informesAnuales/info rmes/VII_Informe_Anual.htm [01/02/2017].

- Delegación del Gobierno para la Violencia de Género (2015): Macroencuesta Violencia Contra la Mujer. Madrid: Ministerio de Sanidad, Servicios Sociales e Igualdad. Versión [online] Disponible en: http://www.violenciagenero.msssi.gob.es/violenciaEnCifras/estudios/colecciones/pdf/Libro_22 _Macroencuesta2015.pdf [01/02/2017].

- Galtung, Johan (2003): Tras la violencia, 3R: reconstrucción, reconciliación, resolución. Afrontando los efectos visibles e invisibles de la guerra y la violencia. Gernika. Bakeaz/Gernika Gogoratuz.

- Gobierno de Andalucía (2007): Ley 13/2007, de 26 de noviembre, de medidas de prevención y protección integral contra la violencia de género en Andalucía. BOE núm. 38, de 13 de febrero de 2008. Versión [online] Disponible en: https://www.boe.es/boe/dias/2008/02/13/pdfs/A0777307785.pdf [01/02/2017].

- Gobierno de Aragón (2007): Ley 4/2007, de 22 de marzo, de prevención y protección integral a las mujeres víctimas de violencia en Aragón. BOE núm. 141, de 13 de junio 2007. Versión [online] Disponible en: https://www.boe.es/boe/dias/2007/06/13/pdfs/A25632-25640.pdf [01/02/2017].

- Gobierno de Canarias (2003): Ley de la Comunidad de Canarias 16/2003, de 8 de abril, de prevención y protección integral de las mujeres contra la violencia de género. BOE núm. 162, de 8 de julio de 2003. BOE núm. 199, de 20 de agosto 2002. Versión [online] Disponible en: https://www.boe.es/boe/dias/2003/07/08/pdfs/A26392-26402.pdf [01/02/2017]. 
- Gobierno de Cantabria (2004): Ley de Cantabria 1/2004, de 1 de abril, integral para la prevención de la violencia contra las mujeres y la protección a sus víctimas. BOE núm.101, de 26 de abril de 2004. Versión [online] Disponible en: https://www.boe.es/boe/dias/2004/04/26/pdfs/A16548-16553.pdf [01/02/2017].

- Gobierno de Castilla-La Mancha (2001): Ley 5/2001, de 17 de mayo de 2001, de las cortes de Castilla-La Mancha, de prevención de malos tratos y de protección a las mujeres maltratadas. BOE núm. 148, de 21 junio 2001. Versión [online] Disponible en: https://www.boe.es/boe/dias/2001/06/21/pdfs/A22038-22041.pdf [01/02/2017].

- Gobierno de Castilla y León (2010): Ley 13/2010, de 9 de diciembre, contra la violencia de género en Castilla y León. BOE núm. 317, de 30 de diciembre de 2010. Versión [online] Disponible en: https://www.boe.es/boe/dias/2010/12/30/pdfs/BOE-A-2010-20072.pdf [01/02/2017].

- Gobierno de Cataluña (2008): Ley 5/2008, de 24 de abril, del derecho de las mujeres a erradicar la violencia machista. BOE, núm. 131, de 30 de mayo de 2008. Versión [online] Disponible en: https://www.boe.es/boe/dias/2008/05/30/pdfs/A25174-25194.pdf [01/02/2017].

- Gobierno de España (2004): Ley Orgánica 1/2004, de 28 de diciembre, de medidas de protección integral contra la violencia de género. BOE núm. 313, de 29 diciembre 2004. Versión [online] Disponible en: https://www.boe.es/boe/dias/2004/12/29/pdfs/A42166-42197.pdf [01/02/2017].

- Gobierno de España (2007): Ley Orgánica 3/2007, de 22 de marzo, para la igualdad efectiva de mujeres y hombres. BOE núm. 71, de 23 de marzo de 2007. Versión [online] Disponible en: https://www.boe.es/boe/dias/2007/03/23/pdfs/A12611-12645.pdf [01/02/2017].

- Gobierno de Extremadura (2011): Ley 8/2011, de 23 de marzo, de igualdad entre mujeres y hombres y contra la violencia de género en Extremadura. BOE núm. 88, de 13 de abril de 2011. Versión [online] Disponible en: https://www.boe.es/boe/dias/2011/04/13/pdfs/BOE-A-20116651.pdf [01/02/2017].

- Gobierno de Galicia (2007): Ley 11/2007, de 27 de julio, gallega para la prevención y el tratamiento integral de la violencia de género. BOE núm. 226, de 20 de septiembre de 2007. Versión [online] Disponible en: https://www.boe.es/boe/dias/2007/09/20/pdfs/A3829838309.pdf [01/02/2017].

- Gobierno de las Islas Baleares (2006): Ley, 12/2006, de 20 de septiembre, para la mujer. Illes Balears. BOE núm. 248, de 17 de octubre 2006. Versión [online] Disponible en: https://www.boe.es/boe/dias/2006/10/17/pdfs/A35830-35830.pdf [01/02/2017].

- Gobierno de La Rioja (2011): Ley 3/2011, de 1 de marzo, de prevención, protección y coordinación institucional en material de vilolencia en La Rioja. BOE núm. 66, de 18 de marzo de 2011. Versión [online] Disponible en: https://www.boe.es/boe/dias/2011/03/18/pdfs/BOE-A2011-4951.pdf [01/02/2017]. 
- Gobierno de Madrid (2005): Ley 5/2005, de 20 de diciembre, integral contra la violencia de género de la Comunidad de Madrid. BOE núm. 52, de 2 de marzo 2006. Versión [online] Disponible en: https://www.boe.es/boe/dias/2006/03/02/pdfs/A08515-08526.pdf [01/02/2017].

- Gobierno de Murcia (2016): Ley 11/2016, de 15 de junio, por la que se modifica la Ley 7/2007, de 4 de abril, para la igualdad entre mujeres y hombres, y de protección contra la violencia de género en la región de Murcia. BOE núm. 167, de 12 de julio de 2016. Versión [online] Disponible en: https://www.boe.es/boe/dias/2016/07/12/pdfs/BOE-A-2016-6648.pdf [01/02/2017].

- Gobierno de Navarra (2002): Ley Foral 22/2002, de 2 de julio, para la adopción de medidas integrales contra la violencia sexista de Navarra. BOE núm. 199, de 20 de agosto 2002. Versión [online] Disponible en: https://www.boe.es/boe/dias/2003/04/25/pdfs/A16062-16064.pdf [01/02/2017].

- Gobierno de Navarrra (2015): Ley Foral 14/2015, de 10 de abril, para actuar contra la violencia hacia las mujeres. Navarra. BOE núm. 107, de 5 de mayo de 2015. Versión [online] Disponible en: https://www.boe.es/boe/dias/2015/05/05/pdfs/BOE-A-2015-4950.pdf [01/02/2017].

- Gobierno de la Comunidad Valenciana (2012): Ley 7/2012, de 23 de noviembre, de la Generalitat, integral contra la violencia sobre la mujer en el ámbito de la Comunitat Valenciana. BOE núm. 297, de 11 de diciembre de 2012. Versión [online] Disponible en: https://www.boe.es/boe/dias/2012/12/11/pdfs/BOE-A-2012-14978.pdf [01/02/2017].

- Gobierno del País Vasco (2013): Plan Director para la coeducación y la prevención de la violencia de género en el sistema educativo del Gobierno Vasco. Vitoria-Gasteiz: Servicio Central de Publicación del Gobierno Vasco. Versión [online] Disponible en: http://www.hezkuntza.ejgv.euskadi.eus/contenidos/informacion/dig_publicaciones_innovacion/ es_conviven/adjuntos/coeducacion/920006c_Pub_EJ_hezkidetza_plana_c.pdf [01/02/2017].

- Gobierno del País Vasco (2005): Ley 4/2005, de 18 de febrero, para la Igualdad de mujeres y hombres del País Vasco. BOE núm. 274, de 14 de noviembre de 2011. Versión [online] Disponible en: https://www.boe.es/boe/dias/2011/11/14/pdfs/BOE-A-2011-17779.pdf [01/02/2017].

- Gobierno del Principado de Asturias (2011): Ley 2/2011, de 11 de marzo, para la igualdad de mujeres y hombres y la erradicación de la violencia de género. Principado de Asturias. BOE núm. 106, de 04 de mayo de 2011. Versión [online] Disponible en: https://www.boe.es/boe/dias/2011/05/04/pdfs/BOE-A-2011-7887.pdf [01/02/2017].

- Observatorio para la Igualdad de Oportunidades (2008): Guía de Coeducación. Síntesis sobre la Educación para la Igualdad de Oportunidades entre Mujeres y Hombres. Madrid: Instituto de la Mujer. Versión [online] Disponible http://www.inmujer.gob.es/observatorios/observIgualdad/estudiosInformes/docs/009-guia.pdf [01/02/2017]. 
- OMS - Organización Mundial de la Salud (1996): "Prevensión de la Violencia: una prioridad en salud pública”. Resolución 49.25 de la 49a Asamblea Mundial de la Salud de 25 de mayo de 1996. Disponible en: http://apps.who.int/iris/bitstream/10665/203955/1/WHA49_R25_spa.pdf [01/02/2017].

- OMS - Organización Mundial de la Salud (1998): "Violencia contra la mujer. Un tema de salud prioritario". Disponible en: http://www.who.int/gender/violence/violencia_infopack1.pdf [01/02/2017].

- ONU (1994): Declaración sobre la eliminación de la violencia contra la mujer. 85 ${ }^{\mathrm{a}}$ sesión plenaria, Asamblea General de Naciones Unidas, 20 de diciembre de 1993. A/RES/48/104, de 23 de febrero de 1994. Versión [online] Disponible en: http://www.un.org/es/comun/docs/?symbol=A/RES/48/104 [01/02/2017].

- ONU (1995): Informe de la Cuarta Conferencia Mundial sobre la Mujer. Celebrada en Beijing de 4 a 15 de septiembre de 1995. Nueva York: Naciones Unidas. Versión [online] Disponible en: http://www.un.org/womenwatch/daw/beijing/pdf/Beijing\%20full\%20report\%20S.pdf [01/02/2017].

- Pérez, Cruz y Gargallo, Bernardo (n.d.): "Sexismo y estereotipos de género en los textos escolares. Addenda a la ponencia IV lectura y género: leyendo la invisibilidad”. Disponible en: http://www.uv.es/genero/_docs/public_edu/sexismo_txtos.pdf [09/02/2017].

- Ruiz-Jarabo, Consuelo; Blanco, Pilar (dir.) (2005): La violencia contra las mujeres. Prevención y detección. Madrid: Díaz de Santos.

- Universidad de País Vasco (2011): "La violencia contra las mujeres y sus causas en Guipúzcoa. Departamento de Política Social de la Diputación Foral de Guipúzcoa”. Disponible en: http://www.bizkaia.eus/gizartekintza/Genero_Indarkeria/blt28/documentos/gipuzkoa_c.pdf [01/02/2017].

- VV.AA. (2011): Igualdad y Prevención de la Violencia de Género en la Adolescencia. Colección Documentos contra la violencia de género. Madrid: Ministerio de Sanidad, Política Social e Igualdad.

- Walker, Leonor (1979): The battered woman. New York: Harper \& Row. 